\title{
Groupe pour L'Avancement de la Microchirurgie Canada (GAM)
}

\author{
Abstracts presented at the \\ 29th Annual Meeting / 29e Réunion annuelle \\ June 25, 2008
}

\section{1}

\section{UTILIZATION OF THE SIEV IN VENOUS CONGESTED DIEP FLAPS. AN OPERATIVE ALGORITHM}

T Hayakawa, E Buchel

University of Manitoba, Winnipeg, Manitoba

PURPOSE: To maximize the benefits of deep inferior epigastric perforator flaps (DIEP) flaps, they should be based on one or two perforators. Harvesting large volumes of tissue on a limited number of perforators occasionally results in flaps with inadequate venous drainage. We routinely use the superficial inferior epigastric vein (SIEV) as a "bail out" for this situation.

METHODS: 200 consecutive DIEP flaps were reviewed.

RESULTS: 10 of the 200 DIEP flaps (5\%) demonstrated clinical venous insufficiency, despite patency of the deep inferior epigastric veins. 9 of the 10 were "hemi flaps" (zones I \& III). One flap included all 4 zones. 8 of the 10 were deemed congested during the initial surgery and an SIEV bailout was immediately performed. 2 were thought only to be "slightly congested" but ultimately required an SIEV bailout when taken back at a later time. The SIEV anastomosis were performed to the following recipients: IMV in 5 patients; retrograde into the distal stump of an IMV in 1; a local unnamed lateral chest wall vein in 2 ; to a branch of the thoracoacromial vein in 1 ; and the thoracodorsal vein using a vein graft in 1 . There was no total or partial flap necrosis.

CONCLUSIONS: The dominant venous drainage pattern of the lower abdominal skin and fat is through the superficial venous system (SIEV). DIEP flaps may have problems with venous drainage due to inadequate collaterals or the presence of valves between the deep and superficial venous systems. Utilizing the SIEV can salvage a DIEP flap when this situation occurs. We present a structured algorithm for managing this situation.

\section{2}

\section{LASER-ASSISTED ICG ANGIOGRAPHY (LA-ICGA); APPLICATIONS IN MICROSURGICAL BREAST RECONSTRUCTION \\ MC Samson, MI Newman \\ Cleveland Clinic, Fort Lauderdale, Florida, USA}

PURPOSE: The ability to confirm perforator flap perfusion is a key factor in intraoperative decision-making. LA-ICGA has been used for decades in the diagnosis and treatment of retinal disorders and recently successfully by cardiac, urologic and liver transplant surgeons for its ability to provide unparalleled, real time vascular images. We report here the initial experience with the application of this technology in perforator flap surgery for breast reconstruction.

METHODS: Following IRB approval, LA-ICGA was introduced into our D.I.E.P. protocol. In a prospective fashion, LA-ICGA was performed prior to harvest and following microsurgical anastomosis. Examined and imaged prior to harvest and following inset were the arterial and venous anastomoses, the vascular pedicles and the perfusion of the flaps through both adipose tissue and skin.

RESULTS: Ten (10) DIEP flaps were performed on eight (8) female breast cancer patients. Flap survival was $100 \%$. LA-ICGA imaging helped to identify intraoperatively: one flap with inadequate subdermal plexus perfusion leading us to débride the distal portion of the flap prior to harvest; one flap with inadequate venous return leading us to seek additional perforators prior to harvest; and, one flap in which inadequate perfusion of a mastectomy flap was identified leading us to débride the marginal tissue prior to final inset.

CONCLUSIONS: LA-ICGA appears to be a valuable adjunct in perforator flap surgery. It can be used to evaluate perfusion prior to harvest and following anastomosis. As additional data is collected and analyzed, the ability to interpret findings will develop. A multicenter trial is recommended to evaluate the effect of this new technology on clinical outcome.

\section{3}

\section{RETROGRADE INTERNAL MAMMARY VESSELS IN BREAST RECONSTRUCTION: AN EFFECTIVE SOLUTION TO LIMITED ANTEGRADE VESSEL FLOW SITUATIONS.}

\section{E Buchel, T Hayakawa}

University of Manitoba, Winnipeg, Manitoba

PURPOSE: The Internal Mammary vessels are the preferred recipient vessels for free breast and chest wall reconstruction at our institution. In a limited number of cases, antegrade flow through the IMA and IMV could not be established. The purpose of this lecture is to highlight the technique and results of using the IMA and IMV in a retrograde fashion.

METHODS: A retrospective chart review was performed using our Microsurgical Database.

RESULTS: 606 Free Breast reconstructions were reviewed between July 2004 and December 2007. 3 retrograde IMA vessels were utilized. 6 retrograde IMV vessels were utilized. All Flaps survived. Two flaps sustained clinically significant fat necrosis requiring revisional surgery. One patient required post-operative anticoagulation.

CONCLUSIONS: The internal mammary vessels are used in all cases of free breast reconstruction at our institution. In a limited number of cases ( $1.48 \%, 9 / 606)$ antegrade flow could not be established in either the artery or vein. In these cases, retrograde flow through the internal mammary vessels was established successfully, thus avoiding further recipient vessel dissection. While not common, retrograde internal mammary vessel free breast reconstruction is a viable solution to a difficult intra-operative problem.

\section{4}

\section{SCIGAP FLAP: BREAST RECONSTRUCTION}

JS Williamson, C Bowmen, D Williamson

Kelowna, British Columbia

PURPOSE: To assess the clinical utility of the septocutaneous IGAP perforator flap in autogenous breast reconstruction.

METHOD: A retrospective analysis of 15 scIGAP flap breast reconstructions performed in 9 patients was performed. Clinical settings, flap design, operative experience, functional and aesthetic outcome, and donor morbidity were reviewed in an effort to further delineate the abilities and limitations of this flap.

RESULTS: Three unilateral and six bilateral scIGAP breast reconstructions were performed between 2003 \& 2007. Fourteen flaps survived. All patients achieved their desired mound reconstructions, though one patient required a further flap procedure and two required prostheses to attain this. Donor site morbidity increased with aggressive skin \&/or subcutaneous harvests. Immediate bilateral reconstructions with multiple intra-operative repositionings led to substantial operative times.

CONCLUSIONS: The septocutaneous IGAP perforator flap, while not widely described, is a viable "alternative flap" for autogenous breast reconstruction. Its attributes of ease of harvest, pedicle length, ability to neurotize, and acceptable donor deformity must to balanced against the limitations of its physical attributes, potential donor morbidity and need for intra-operative repositioning in considering its indications in each patient. 


\section{5}

\section{AUGMENTATION OF FREE AUTOLOGOUS BREAST} RECONSTRUCTION

\section{S Olivier, T Hayakawa, E Buchel}

University of Manitoba, Winnipeg, Manitoba

PURPOSE: Free Perforator flap breast reconstruction is the standard of care at our institution. In selected cases, the volume is inadequate for symmetry or the patient desires an increased breast size In these cases, augmentation of the autogenous reconstruction was performed. We present our indications, technique, timing and outcomes of implant augmentation of free autologous breast reconstruction.

METHOD: This is a retrospective chart review of our Microsurgery Database.

RESULTS: 1006 free tissue transfers were reviewed in the database between July 2004 and December 2007. Of these 484 patients underwent 606 free breast reconstructions. 13 autologous breast reconstruction underwent augmentation. No free flaps were compromised. 1 augmentation needed to be removed secondary to an implant infection not amendable to medical management. 2 patients underwent a revision surgery for symmetry or shape modification.

CONCLUSIONS: Autologous tissue breast reconstruction is adequate for size and symmetry in the vast majority of cases. If expectations are not met, the addition of a sub-flap augmentation can result in the desired outcome.

\section{6}

\section{PULMONARY EMBOLISM IN BREAST RECONSTRUCTION PATIENTS}

\Giuffre, E Buchel, T Hayakawa

University of Manitoba, Winnipeg, Manitoba

PURPOSE: Pulmonary embolism (PE) is a known complication of free tissue transfer in breast reconstruction patients. There is no literature outlining the incidence and risk factors in this group of patients. The purpose is to identify the incidence of PE in breast reconstruction patients who have undergone free tissue transfer.

METHODS: All patients undergoing free flap breast reconstruction were reviewed. Patient demographics, comorbidities, diagnosis, type of flap, perioperative fluid volume, duration of anesthesia, symptoms, imaging, treatment, and complications were recorded.

RESULTS: The incidence of PE was 12/484 (2.5\%). No patients had previous venous thromboembolism (VTE). Patients presented 3-20 days postoperatively with decreased oxygen saturation, tachypnea, shortness of breath and tachycardia. The diagnosis was confirmed with a spiral CT scan. Perioperative DVT prophylaxis protocol consisted of: preoperative placement of SCDs and TED stockings continued until third postoperative day; intraoperative intravenous Toradol continued until 72 hours; postoperative SC heparin; and mobilization beginning on the 2nd post-operative day. Patients with PE were treated with intravenous Heparin and discharged on Dalteparin or Coumadin. One massive PE resulted in a fatality. There were also 2 flap failures in this group. We address correlations between PE and body mass index (BMI), cancer stage, perioperative fluid administration and operative times.

CONCLUSIONS: We report a $2.5 \%$ incidence of PE in patients undergoing free flap breast reconstruction. This group is theoretically at high risk given lengthy surgical times, immobility, and a history of malignancy.

\section{7}

\section{SWITCHING TO THE ROUTINE REMOVAL OF THE 4TH COSTAL CARTILAGE FOR INTERNAL MAMMARY VESSELS EXPOSURE IN FREE-FLAP BREAST RECONSTRUCTION: OUTCOME ANALYSIS}

W Menesi, T Hayakawa, E Buchel

University of Manitoba, Winnipeg, MB

PURPOSE: Removal of the 3rd costal cartilage to access the internal mammary (IM) vessels is the preferred choice for many surgeons when performing free-flap breast reconstruction. Careful evaluation revealed a contour deformity in the medial breast and chest in approximately one third of our patients when this approach was used. We have therefore switched to removing the 4 th costal cartilage for IM vessels exposure. The purpose is to evaluate the advantages, difficulties, and complications in patients who underwent a 4th costal cartilage approach.

METHODS: A retrospective review from January 2007 to December 2007.

RESULTS: A total of 131 free flaps were reviewed in 100 patients. The 4th costal cartilage was used for IM vessel access for 75 flaps in 58 patients. The 3 rd costal cartilage was used for 56 flaps in 42 patients. At the level of the $3 \mathrm{rd}$ interspace the mean diameter of the venous coupler used was $2.1 \mathrm{~mm}$ compared to $2.0 \mathrm{~mm}$ at the 4 th interspace. Flap loss ( $1.5 \mathrm{vs} .1 .12$ $\%)$, venous thrombosis ( 2.24 vs. $0.75 \%)$, hematoma ( 0.56 vs. $1.5 \%)$, and fat necrosis ( 1.5 vs. $1.12 \%)$, were comparable between the $3 \mathrm{rd}$ and 4 th costal cartilages groups respectively. However, there were no contour deformities in the 4th costal cartilage group. In only one case did the 3rd costal cartilage need to be removed due to inadequate venous size at the level of the 4th.

CONCLUSIONS: We found no increased flap complications when the 4th interspace was used for free flap anastomosis instead of the $3 \mathrm{rd}$. Its use eliminates any unpleasant contour deformities that occur with 3rd costal cartilage removal.

\section{8}

\section{DOES PERFORATOR CHOICE AFFECT THE OUTCOME OF DIEP BREAST RECONSTRUCTION?}

\section{G Al Thubaiti, S Rabah, T Hayakawa, E Buchel}

University of Manitoba, Winnipeg, MB

PURPOSE: The DIEP flap has become popular secondary to its decreased donor morbidity. Morbidity is reduced by harvesting a minimum number of perforators from the deep inferior epigastric artery. The use of a single perforator has not gained wide acceptance due to the concerns of vascular insufficiency of the flap. We report our experience with limited perforator harvesting on our DIEP flap cases

METHOD: A single surgeon retrospective review of 628 free flaps from July 2004 to December 2007. 221 DIEP flaps were used for Breast reconstruction. The intraoperative perforator choices were reviewed. A comparative study was done between the perforator choice and the complications which included: fat necrosis; partial or total flap loss; and donor site complications.

RESULTS: Of 221 consecutive DIEP flaps the most common choice was a single medial row perforator $(\mathrm{n}=120,54 \%)$, followed by double medial row perforators $(n=41,18 \%)$ and a single lateral perforator $(n=32,14.4 \%)$. Fat necrosis rate were as follows; $10 \%$ in the single medial perforator group; $4.3 \%$ in the double medial perforator group; $9.4 \%$ in the single lateral perforator group; and $4.6 \%$ in the double lateral perforator group.

CONCLUSION: In our experience, the most common perforator choice was a single medial row, followed by double medial row perforators and single lateral row perforator.In total, $68.4 \%$ of the flaps were harvested on a single perforator. Although there was a trend in the single perforator groups to have higher incidence of fat necrosis $9.8 \%$, versus $4.6 \%$ in the double perforators groups, this difference was not significant ( $\mathrm{p}$ value $=0.098$ ).

\section{9}

SURGICAL OUTCOMES OF VRAM VS. THIGH-BASED FLAPS FOR RECONSTRUCTION OF PELVIC AND PERINEAL CANCER RESECTION DEFECTS

\section{R Nelson, Butler CE}

Dalhousie University, Halifax, Nova Scotia; M.D. Anderson,

Houston, Texas, USA

PURPOSE: To directly compare the surgical outcomes of cancer patients undergoing immediate reconstruction of APR and PE defects with VRAM vs. thigh-based flaps.

METHOD: A retrospective review of all consecutive patients undergoing APR or PE for primary colorectal or urogynecologic malignancy and immediate reconstruction with a VRAM or thigh-based flap at M.D. Anderson between 1993-2007 was performed. Patient characteristics and surgical outcomes were compared between patients in the VRAM and thigh flap groups, and logistic regression was used to identify predictive/protective factors for donor and recipient-site complications. RESULTS: 133 patients (VRAM, $\mathrm{n}=114$ and thigh, $\mathrm{n}=19$ ) were included in the study. The mean follow-up was 23 months. There were no differences in patient demographics, comorbidities, tumor pathology, disease 
stage or length of follow-up between groups. Patients in the thigh group had a significantly greater incidence of donor site complications including cellulitis [26\% vs. $6 \%$ ] and recipient site complications (cellulitis [21\% vs. $4 \%$ ], pelvic abscess formation [32\% vs. $6 \%$, and major wound dehiscence [ $21 \%$ vs. $5 \%]$ ). There was no increase in abdominal wall complications including dehiscence, hernia, seroma, or infection in the VRAM group despite flap harvest from the abdominal wall. Logistic regression analysis identified obesity $(\mathrm{BMI} \geq 30)$ to be the only independent predictor for major donor site complications (odds ratio [OR], 3.3) and previous abdominal surgery to be a predictor for major recipient site complications (OR, 3.5).

CONCLUSIONS: Primary reconstruction of APR and PE defects with VRAM flaps results in fewer major donor and recipient site complications than thigh-based flaps without increased abdominal wall morbidity and, if available, should be strongly considered as the primary flap option for these defects.

\section{0}

\section{MICROSURGICAL AUTOTRANSPLANTATION OF LYMPH NODES AND THE PREVENTION OF EXPERIMENTAL LYMPHEDEMA}

D Tobbia, J Semple, M Johnston University of Toronto, Toronto, Ontario

PURPOSE: Lymphedema in breast cancer patients continues to represent a distressing consequence of axillary node resection. We propose the novel concept that the lymph node itself has important functions in maintaining fluid balance and it is the removal of the lymph node rather than damage to the lymphatic vessels that could be the key to initiate lymphedema. The purpose of this study was to develop an experimental approach to quantify lymphedema and lymph transport after lymph node excision in sheep. We examined whether the re-implantation of a vascularized lymph node would prevent edema and restore lymphatic function.

METHODS: Lymph transport was quantitated in the popliteal lymphatic system of sheep by injecting radioactive Human Serum Albumin (HSA) into one of the prenodal lymph vessels. The recovery of the tracer in plasma was measured at 8,12 and 16 weeks after nodal excision. These values were compared with similar time points in intact limbs (control) and limbs that received a vascularized autologous popliteal lymph node transplant. Edema in each limb was quantified from a standardized circumferential measurement of the hind limb.

RESULTS: Following lymph node removal, the control limbs became progressively more edematous up to three days following nodectomy (increase of $33.8 \%$ relative to pre-surgical state). Thereafter, the edema improved but did not resolve completely in the majority of animals even at 16 weeks post surgery. Assessment of the mass transport of the HSA revealed recoveries at 8,12 and 16 weeks $(\%$ injected/hr) of $10.6 \pm 1.5$ $(\mathrm{n}=7), 14.4 \pm 1.0(\mathrm{n}=7)$ and $13.9 \pm 1.0(\mathrm{n}=6)$ respectively compared with $19.3 \pm 2.2$ in node-intact limbs $(n=8)$. Following autologous lymph node transplantation, the preliminary results were variable with recoveries of 18.2,15.3 and 16.1 at 8 weeks and 20.7 and 12.6 at 12 weeks. Failed microvascular anastomosis of the node may have contributed to low recoveries in some sheep. Edema in two of the animals at 8 and one of the animals at 12 weeks post microvascular node transplant resolved completely, incidentally these were also the animals that showed mass transport rates similar to the control group.

CONCLUSION: We developed a new quantitative method to assess the physiological impact of lymph node excision in a sheep lymphedema model. Even up to 16 weeks after nodal resection, lymphatic function had not been restored to node-intact levels.

Micro vascular re-implantation of an autologous lymph node following nodectomy in the limb of a sheep may have the potential to prevent the formation of chronic edema and help restore lymphatic transport function.

\section{1}

THE USE OF LOCAL FLAPS AS A SOLUTION FOR ORAL INCOMPETENCE FOLLOWING HEAD AND NECK FREE FLAP RECONSTRUCTION

M Choi, EW Buchel, T Hayakawa

University of Manitoba, Winnipeg, Manitoba

PURPOSE: Surgical management of extensive head and neck tumors often requires full thickness resection of a segment of the muscular component of the lower lip or results in an element of deinnervation. Despite successful free flaps to reconstruct intraoral anatomic and functional components, lower lip function is often compromised. Secondary reconstructive options to reconstitute sphincter function, sensation, and overall oral competence are limited. The purpose of this study is to describe the utility and functional outcomes of local flaps in the correction of oral incompetence following resection and free flap reconstruction in head and neck cancer patient.

METHOD: Chart review and review of the literature.

RESULTS: Four patients underwent composite resection of head and neck tumors involving partial lower lip sacrifice or deinnervation and successful free flap reconstruction. All patients had interruption of facial vessel continuity during neck dissection or as recipient vessels for free flap transfer. All patients had chronic oral incontinence following healing of their wounds and completion of radiation therapy. Local lip advancement flaps based on the facial artery angiosomes were used to restore oral competence. Karapandzic flaps were utilized in 2 patients, a Johansson step flap in one, and a modified lower lip advancement flap in one.

CONCLUSIONS: Oral incompetence following free flap reconstruction of head and neck tumors can effectively be reconstructed using conventional local lip advancement flaps, despite irradiation and previous sacrifice of the facial artery.

\section{2}

\section{ANATOMICAL BASIS TO THE PERONEAL ARTERY PERFORATOR FLAP (PAP)}

\section{R Ahmadzadeh, M Tang, SF Morris}

\section{Dalhousie University, Halifax, Nova Scotia}

PURPOSE: The purpose of this study is to comprehensively evaluate the anatomical basis of the peroneal artery perforator flap and to introduce anatomical landmarks to facilitate flap dissection.

METHODS: Six fresh cadavers underwent intra-arterial injection with a lead oxide and gelatine preparation. The integument of the leg was dissected $(n=11)$. Cutaneous perforators of the peroneal artery were identified. Their type (septocutaneous vs. musculocutaneous), location, course, and size were documented by dissection, angiography and photography. The surface areas of cutaneous territories and perforator zones were measured and calculated.

RESULTS: The average number of cutaneous perforators $\geq 0.5 \mathrm{~mm}$ in diameter, arising from the peroneal artery is $5 \pm 1$. Thirty percent of these perforators are septocutaneous and $70 \%$ are musculocutaneous. Their average internal diameter is $0.8 \pm 0.3 \mathrm{~mm}$. The average pedicle length from the deep fascia is $36 \pm 20 \mathrm{~mm}$. The average cutaneous vascular territory supplied by the peroneal artery is $172 \pm 42 \mathrm{~cm}^{2}$, which comprises $19 \pm 5 \%$ of the leg integument (measured from the femoral epicondyles to the malleoli). The average perforator zone is $36 \pm 11 \mathrm{~cm}^{2}$. The peroneal artery perforators are found along the distal three fourths of a line drawn from the lateral femoral epicondyle to the Achilles tendon; most of the distal perforators are septocutaneous.

CONCLUSION: This study illustrates the surgical anatomy of the peroneal artery perforator flap. Additional clinical studies can take advantage of the reliable size and consistency of these perforators to use them in pedicled[1] or free flaps[2] in a variety of reconstructive cases.

\section{P-1}

\section{RECONSTRUCTION FOLLOWING TRAUMATIC SEGMENTAL SCALP AVULSION: THE USE OF REPLANTATION AND COMPOSITE GRAFT: A CASE REPORT}

\section{Dickson, A Thoma}

McMaster University, Hamilton, Canada

Total scalp avulsion is a devastating and disfiguring injury. Microvascular replantation in scalp reconstruction following traumatic avulsion was first described by Miller et $\mathrm{al}^{1}$ in 1976 and has since become the mainstay and standard of care in such injuries. Prior to the advent of microvascular scalp reconstruction such injuries were treated by skin grafting the degloved calvarium. We present a case of a 54-year old male with a total occipital scalp avulsion ( 30\% scalp surface area). The avulsed skin was in two separate segments and included the periosteum. The larger segment was microsurgically replanted with two venous anastomoses, one being an interposi- 
tional vein graft, and a single arterial anastomosis to the occipital artery. The smaller segment of avulsed scalp had no identifiable arteries or veins available for anastomosis. This smaller segment was replaced as a composite graft. The composite graft did not survive; however, it became adhered to the raw bone and acted as a biological dressing which protected the underlying defect while it underwent healing by secondary intention. As the mummified dry scalp segment contracted it resulted in expansion of the surrounding scalp, including the microvascularly replanted segment of scalp. This is the first reported case of its kind and presents a unique solution to a rare and difficult problem.

\section{P-2}

\section{PERIOPERATIVE MICROSURGICAL FREE FLAP MANAGEMENT: CANADIAN PLASTIC SURGICAL PERSPECTIVE}

\section{AN Seal, S Macadam, DJ Courtemanche, M Hill}

\section{University of British Columbia}

PURPOSE: The purpose of this study is to investigate the practice of Canadian Plastic Surgeons with respect to perioperative care of patients undergoing microsurgical free flap reconstruction. The study focuses on management of anticoagulation and vascular physiology.

METHOD: Canadian Plastic Surgeons were contacted though the Canadian Society of Plastic Surgery active email list and asked to complete an anonymous online survey focusing on pre-, intra-, and post-operative parameters relating to microsurgical flap management.

RESULTS: There was a response rate of $27 \%$ (67 of 248), with an estimated 1937 combined microsurgical free flaps per year in Canada. Fellowship trained microsurgeons do more cases per year, (mean 36 vs. 12, $p=0.003$ ). There is no difference in the number of cases performed by location - academic vs. community practice. Post-operatively, $18 \%$ of plastic surgeons use low molecular weight heparin, 24\% Dextran, and 55\% aspirin in their anticoagulation regimes. There is no difference in the pattern of practice with respect to pre-, intra-, or postoperative heparin, ASA or dextran vs. either volume of cases, fellowship training, or location of practice. Surgeons who do fewer cases are more likely to use a warm room postoperatively $(\mathrm{p}=0.004)$. Appearance and Doppler are the most common post-operative modalities used for monitoring $(100 \%$ and $70 \%$ respectively)

CONCLUSIONS: There is no current consensus in the literature on guidelines or recommendations for postoperative pharmacologic or general patient care of patients undergoing microsurgical free flap reconstruction. Much diversity in the use of pharmacologic agents in microsurgery remains.

\section{P-3}

\section{NON-BREAST RECONSTRUCTION USING THE FREE DIEP} FLAP

\section{Q Chivers, G Al Thubaiti, T Hayakawa, E Buchel}

University of Manitoba, Winnipeg, MB

PURPOSE: A long vascular pedicle, large skin island, ability to include fascia and primary donor site closure are some of the most significant advantages of the DIEP flap. These advantages afford the DIEP flap the ability to reconstruct large soft tissue defects where other flaps would result in vein grafting and or donor site skin grafting. We report our experience using this flap for general reconstructive purposes excluding the breast.

METHODS: This was a retrospective review of our Microsurgery Database.

RESULTS: 1006 microsurgical cases were reviewed from July 2004 until December 2007. 15 consecutive cases of non-breast surgical defects were reconstructed with DIEP flaps between July 2003 and December 2007.

Surgical defects were secondary to: sarcoma resection on the shoulder, chest wall, popliteal fossa, and lower extremity (4); SCC resection of the tongue and oropharynx (3); advanced Breast cancers with chest wall and rib resections (3); BCC of the back (1); sacral ulceration (1); Melanoma resection of lower extremity (1); electrical burns to the neck (1); diabetic foot ulcer (1).

Recipient vessels included the: facial artery (4), internal mammary (4), gluteal vessels (2), inferior geniculate (2), posterior tibial vessels (2), thoracoacromial vessels (1).

One flap failure occurred secondary to fistula formation, subsequent wound dehiscence and arterial anastomosis disruption. Venous thrombosis occurred in one flap requiring revision. Partial flap necrosis occurred twice, and abdominal wounds once.

Three patients required secondary procedures for re-contouring of their flaps.

CONCLUSIONS: The DIEP flap is a reliable flap that can be used when a large skin paddle or long vascular pedicle is needed and primary closure of the donor site is desirable.

\section{P-4}

ANTICOAGULATION IN DIGITAL REVASCULARIZATION \& REIMPLANTATION MICROSURGERY: AN ANALYSIS OF BENEFICIAL \& DETRIMENTAL EFFECTS

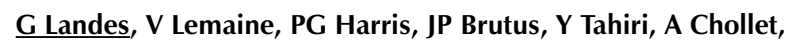
A Dionyssopoulos, A Nikolis

Universite de Montreal

PURPOSE: The aim of the study is to better establish the efficacy and risks associated with three different thromboprophylaxis regimens commonly used in digital replantation microsurgery.

METHODS: All revascularized or reimplanted digits treated at a specialized replantation center over a three year period were included. Type, dosage and duration of medication administration for thromboprophylaxis were evaluated. Primary endpoints were i) the proportion of successfully replanted digits prior to discharge; ii) the incidence of complications in the post-operative period.

RESULTS: Between April 2004 and March 2007, 233 digits were managed by the reimplantation team ( 147 revascularization and 86 reimplantation procedures). More than $98 \%$ of the digits received a post-operative thromboprophylaxis regimen: $50,0 \%$ received aspirin $80 \mathrm{mg}$ once daily plus subcutaneous heparin 5000 UI twice daily (group A; $n=115$ ), 35.7\% received aspirin $80 \mathrm{mg}$ once daily plus IV heparin (group B; $\mathrm{n}=82$ ), and $14.3 \%$ received two weeks of aspirin $80 \mathrm{mg}$ once daily (group C; $\mathrm{n}=33$ ). Success rates at discharge were as follows: group A 94.8\%, group B $89.0 \%$, and group C $87.9 \%(p=0.24)$. The incidence of short term complications in groups A, B, and C were $24.3 \%, 47.6 \%$, and $27.3 \%$ respectively $(\mathrm{p}=0.002)$. Controlling for age, smoking status and the intervention performed, the type of anticoagulation regimen was not significantly related to success rates, whereas digits on IV heparin had a 2.7 times greater risk of complications than digits on subcutaneous heparin (95\% CI, 1.1-6.9).

CONCLUSIONS: Important discrepancies were outlined in the administration of anticoagulation regimens in replantation microsurgery. When considering short-term outcomes following digital reimplantation and revascularization interventions, no significant survival differences were identified based on the type of regimen used. Digits receiving IV heparin had a significantly greater risk of post-operative complications than those receiving subcutaneous heparin. 